\title{
COMPARACIÓN DE DOS TÉCNICAS QUIRÚRGICAS DE LA CESÁREA
}

Laritza Rodríguez, M.D. *, Angel David Miranda, M.D.**, Héctor A. González G., M.D. **

\section{Resumen}

Estudio clínico aleatorio que compara dos técnicas de cesárea en pacientes del Servicio de Obstetricia del Hospital de San José (Bogotá), durante el período comprendido entre enero de 1998 y junio de 1999. De las pacientes con indicación de cesárea, se seleccionaron aleatoriamente 100 que ingresaron a dos grupos: al grupo $1(n=50)$, se le realizó la técnica tradicional (Hhisterorrafia en dos planos con cierre de ambos peritoneos) y en el grupo 2 ( $n=50$ ), la técnica en monoplano (histerorrafia en un plano sin cierre del peritoneo visceral ni parietal). Se evaluaron el tiempo quirúrgico empleado, el sangrado intraoperatorio, la cantidad de material de sutura utilizado, el dolor y las complicaciones en el postoperatorio inmediato. El promedio de edad fue de 25,48 \pm 5,5 años en el grupo 1 (G1) y de 27,5 \pm 7,4 años en el grupo 2 (G2), (p= 0,38 NS). En el grupo 1, 37,5\% eran primigestante y $34,3 \%$ en el grupo 2 ( $p=0,8$ NS). Se encontraron diferencias estadísticamente significativas entre los dos grupos: El grupo 2 (G2) mostró reducción del tiempo quirúrgico (G2: 30,3 $\pm 5,6$ minutos vs $\mathrm{G1:}$ 42,7 \pm 7,3 minutos, $\mathrm{p}<0.01$ ), disminución del sangrado intraoperatorio ( G2: $337 \pm 97$ c.c. vs G1: $475 \pm 54$ c.c., p<0.001), menor cantidad de suturas absorbibles empleadas en el G2: Mediana de 3, significativamente menor que la mediana del G1=5. La mediana de la intensidad del dolor postoperatorio referido por las integrantes del G2 fue de 5, significativamente menor que en el G1, donde fue de 6 $(\mathbf{p}<0.01)$. No se encontraron diferencias con respecto al promedio de horas de estancia hospitalaria y no se presentaron complicaciones postoperatorias. Se sugiere continuar el estudio para realizar el seguimiento de las pacientes y determinar las complicaciones a largo plazo, así como su impacto sobre la morbilidad y el futuro obstétrico.

\section{Introducción}

Según las estadísticas de la institución, la cesárea es el procedimientoquirúrgicoqueconmayor frecuenciaserealiza en el área de ginecología y obstetricia, y por lo tanto es un campo interesante de estudio comparar dos técnicas quirúrgicas y encontraruna que reportemayores beneficios paralas pacientes intervenidasy paralasmismas instituciones.

En la mayoría de los centros de atención obstétrica, dentro de los protocolos de manejo se practica la técnica tradicional de cesárea, que consiste en realizar la histerorrafia en dos planos con cierre de peritoneo visceral y parietal (1).

Existen descritas otras técnicas dentro de las cuales llamala atención aquella en la cual se realiza histerorrafia en un soloplano, sin cierre de peritoneos (2,3), ya que, según los reportes publicados en la literatura mundial esta técnica ofrece aspectos favorables pues disminuye el tiempo quirúrgico, es menor la cantidad de suturas empleadas, provoca menos dolor postoperatorioy reduce laformaciónde adherencias $(2,3,4,5,6,7,8,9)$.

* Servicio de Obstetricia, Hospital de San José, Bogotá

**Fundación Universitaria de Ciencias de la Salud, Hospital de San José, Bogotá
Quienes abogan por esta técnica justifican que lahisterorrafia en un solo plano permite el afrontamiento de los bordes de la incisión (histerotomía) de manera tal, que el mecanismo de cicatrización se hace más fisiológico $(2$, $3)$. Por otra parte, el no cierre del peritoneo ha demostrado que no es relevante al realizar un cierre quirúrgico óptimo y por el contrario ofrece beneficios ya que la formación de adhe-rencias es menor $(4,5,6,7,10)$, lo que sería fundamental en el futuro obstétrico de las pacientes (10) y además, es menor el dolor postoperatorio referido por los pacientes $(7,11,12)$.

Este estudio participa de la tendencia actual en la que se busca la técnica quirúrgica «ideal», es decir aquella que tiene como propósito reducir al mínimo el trauma tisular, optimar el control hemostático, reducir el tiempo operatorio, la morbilidad postoperatoria, la estancia hospitalaria y por lo tanto los costos.

\section{Materiales y métodos}

Con el propósito de comparar dos técnicas de cesárea se realizó un estudio clínico aleatorio en el que participaron setenta pacientes con indicación de cesárea, 
atendidas en el Servicio de Ginecoobstetricia del Hospital San José, entre enero de 1998 y junio de 1999, previa autorización del Comité de Ética e Investigación de la Institución.Las pacientes fueron seleccionadas por medio de un muestreo aleatorio simple, utilizando los registros de aquellas que requerían cesárea el día que el cirujano investigador estaba de turno.

Cada día, dos de las pacientes eran escogidas; si el número de su registro era impar se les asignaba el tratamiento 1 (técnica tradicional) y si el número era par se les asignaba el tratamiento 2 (técnica con histerorrafia en monoplano sin cierre de peritoneos) y el procedimiento se llevóa cabo previo consentimiento informado. Las intervenciones quirúrgicas las realizó un residente de cuarto año y no se tuvo en cuenta el límite de edad de las pacientes para su ingreso al estudio.

Como técnica tradicional se practicó la histerotomía segmentaria transversa con histerorrafia en dos planos: continuo cruzado perforante total e invaginante continuo. Para las suturas se utilizó catgut cromado 1.0 en aguja curva, en tanto que el cierre de peritoneo visceral y parietal se realizó con catgut cromado 2.0.

En la técnica a comparar, aplicada al grupo 2, se realizó histerotomía segmentaria transversa con histerorrafia en un solo plano: continuo cruzado perforante total, sin cierre de peritoneos visceral ni parietal.

Fue permitida la colocación de puntos en equis para controlar hemostasia cuando no era suficiente con un solo plano de histerorrafia, pero si superaba el número de tres se consideraba fracaso de la técnica; es importante mencionar que ninguna paciente fue excluida del estudio por esta causa (11).

El material de sutura empleado fue el mismo que se utilizó en el grupo 1. La incisión en la piel se decidió según cada caso en particular, sin ser limitante para el estudio.

Para contabilizar el tiempo quirúrgico se tomó como tiempo cero el momento de la incisión en la piel y como tiempo final la terminación de la sutura intradérmica, y fue medido en minutos. La cuantificación del sangrado trató de establecerse en forma objetiva, teniendo en cuenta el volumen recolectado en el reservorio de la succión medido en $\mathrm{mL}$ y el recuento de compresas empapadas.

La intensidad del dolor se evaluó a las doce horas del postoperatorio aplicándole a las pacientes la escala verbal numérica modificada de Read y colaboradores, que va de 0 ( $\sin$ dolor) hasta 10 ( peor dolor imaginable).

El esquema analgésico postoperatorio fue igual en ambos grupos: una ampolla de diclofenaco sódico por vía intramuscular al finalizarel procedimiento quirúrgico y luego cada 8 horas.
La cantidad de suturas empleadas era informada por la instrumentadora y registrada en la descripción quirúrgica. La información fue recolectada a través de un formulario que diligenciaba el cirujano, obteniendo los datos de la historia clínica y de entrevista directa con las pacientes en el pre y el postoperatorio.

Dentro de los métodos estadísticos utilizados, para detectar la existencia de diferencias significativas en las variables edad, tiempo quirúrgico y sangrado intraoperatorio, se utilizó el análisis de varianza a una vía paramétrica si cumplía con los supuestos (variabilidad homogénea entre los grupos: prueba de Bartietty los supuestos de la distribución normal).

En caso de no cumplirse los supuestos antes mencionados, se utilizó un análisis de varianza no paramétrico (Kruskall-Wallis), que también se usó en la evaluación del dolor postoperatorio y la cantidad de suturas empleadas. Para el análisis de las indicaciones quirúrgicas fue utilizada la prueba Ji-cuadrado de Pearson; dichas pruebas estadísticas se evaluaron a un nivel de significancia de $5 \%$ y $1 \%$ (NS: No significativo).

\section{Resultados}

Se analizaron y compararon las variables edad, paridad, indicación de cesárea, tiempo quirúrgico, cantidad de suturas empleadas, dolor postoperatorio y sangrado intraoperatorio en cada uno de los grupos; en el grupo 1 $(\mathrm{n}=50)$ se practicó la técnica tradicional de cesárea y en el grupo $2(n=50)$, la técnica con histerorrafia en monoplano sin cierre de peritoneos.

El promedio de la edad \pm DE en el primer grupo fue de 25,48 $\pm 5,5$ años y en el segundo de 27,5 $\pm 7,4$ años ( $\mathrm{p}=0,38$, NS), con un rango mínimo de 16 y máximo de 40 años para ambos grupos. Con respecto a la paridad no se encontraron diferencias significativas, en el grupo $137,1 \%$ correspondió a primigestantes y 34,3\% en el grupo 2( $\mathrm{p}=0,8$, NS). En las multigestantes, la distribución de acuerdo con las cesáreas previas fue similar en ambos grupos; una cesárea previa: 15 pacientes (30\%); dos cesáreas previas: 3 pacientes $(8,6 \%)$; tres cesáreas previas: 1 paciente en cada grupo $(2,9 \%)$.

El porcentaje de pacientes, distribuidas de acuerdo con la indicación quirúrgica para la cesárea se muestra en la tabla 1.

Como se señaló con anterioridad, no se encontraron diferencias significativas desde el punto de vista estadístico en el análisis de las variables edad, paridad o tipo de indicación quirúrgica. Esto permitió hacer una evaluación específica de las variables de interés, mencionadas a continuación. 
Tabla 1.

\begin{tabular}{|c|c|c|c|c|c|}
\hline \multicolumn{6}{|c|}{ Distribución de las indicaciones para la cesárea en cada grupo de pacientes } \\
\hline \multirow[t]{2}{*}{ INDICACIONES } & \multicolumn{2}{|c|}{ GRUPO $1 \mathrm{n}=50$} & \multirow{2}{*}{$\begin{array}{c}\text { GRUPO } \\
\mathrm{N}\end{array}$} & \multirow{2}{*}{$\begin{array}{l}n=50 \\
\%\end{array}$} & \multirow[b]{2}{*}{$\mathrm{p}$} \\
\hline & $\mathrm{N}$ & $\%$ & & & \\
\hline Cesárea anterior & 29 & 40,1 & 29 & 40,1 & $1, \mathrm{NS}$ \\
\hline DCP (Desproporción céfalo-pélvica) & 9 & 18 & 9 & 18 & $1, \mathrm{NS}$ \\
\hline HIE (Hipertensión inducida por el embarazo) & 2 & 4 & 4 & 8 & $0,6 \mathrm{NS}$ \\
\hline Oligoamnios severo & 2 & 4 & 2 & 4 & $0,6 \mathrm{NS}$ \\
\hline Podálico & 4 & 8 & 2 & 4 & $0,6 \mathrm{NS}$ \\
\hline Sufrimiento fetal agudo & 4 & 8 & 4 & 8 & $0,7 \mathrm{NS}$ \\
\hline
\end{tabular}

El promedio del tiempo quirúrgico \pm DE para el grupo 2 fue de $30,3 \pm 5,6$ minutos, significativamente inferior que el encontrado en el grupo 1 , donde fue de $42,7 \pm 7,3$ minutos, p $<0.01$ ( Figura 1).

Para el análisis de valores de intensidad de dolor postoperatorio y sangrado intraoperatorio se utilizó el test de Kruskal-Wallis aplicado para los dos grupos, puesto que el test de Bartlett mostró diferencia entre las varianzas. La mediana de la intensidad del dolor postoperatorio referido por las integrantes del grupo 1 fue de 5 (rango mínimo de 2 y máximo de 7) significativamente mayor que el reportado por las pacientes del grupo 2, donde fue de 6 (rango mínimo de 4 y máximo de 8), p<0,01 (Figura 2).

La mediana de la cantidad de suturas empleadas en el primer grupo fue de 5 (rango de 4 a 6 ), valor significativamente superior al correspondiente para el segundo grupo de pacientes, en quienes fue de 3 (rango de 2 a 3 ), p<0,01.

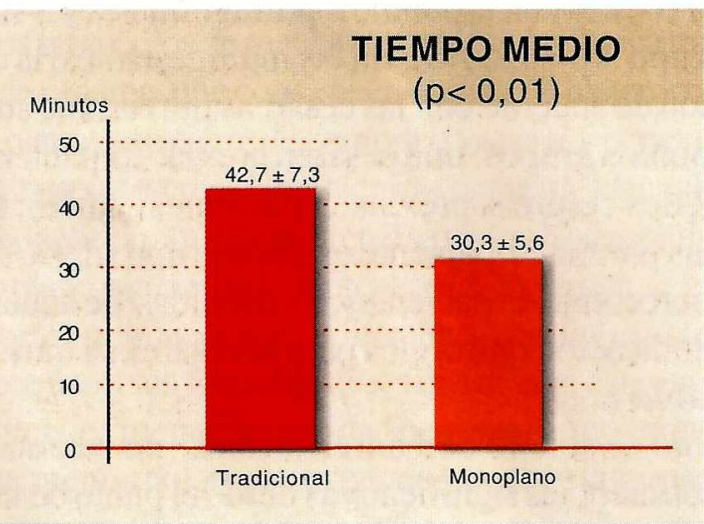

Figura 1. El tiempo quirúrgico empleado en la técnica monoplano fue significativamente menor que el empleado en la técnica tradicional.
En cuanto al sangrado el promedio \pm DE para el grupo 2 fue de $377 \pm 97 \mathrm{~mL}$., con un rango mínimo de 300 mL y un máximo de $600 \mathrm{~mL}$, significativamente mayor que el promedio correspondiente, registrado en el grupo 1 que fue de $475 \pm 54 \mathrm{~mL}, \mathrm{p}<0,01$ (Figura 3).

Por último, el promedio de horas de estancia hospitalaria fue igual en ambos grupos (24 horas) y no se presentaron complicaciones postoperatorias.

\section{Discusión}

Los resultados sugieren que existen diferencias importantes entre las dos técnicas para la realización de la cesárea. Tales diferencias corresponden a la disminución en el tiempo quirúrgico, la intensidad del dolor post-operatorio y el número de suturas empleadas, con la técnica de monoplano, con respecto a la tradicional.

En cuanto a las diferencias entre el volumen de sangrado en ambos grupos, aunque los resultados obtenidos mostraron significado estadístico, no es posible descartar que la medición pudo estar influenciada por un componente subjetivo, por lo que sería importante encontrar una forma de medir con mayor exactitud dicho parámetro en un estudio posterior.

Aunque no fue cuantificado el costo de cada una de las técnicas, puede inferirse que la técnica de la cesárea con histerorrafia en monoplano podría acompañarse de menores costos, ya que requiere una menor cantidad de suturas, así como menos dosis analgésicas durante el postoperatio y, por otra parte, ocasiona una estancia hospitalaria más corta.

La técnica quirúrgica de la cesárea ha tenido múltiples modificaciones desde su inicio, cuandoera una cirugía fatal en la que no se hacía histerorrafia, hasta el año de 1882 fecha en la que Sanger recomendó la sutura inmediata de laincisiónuterina. 


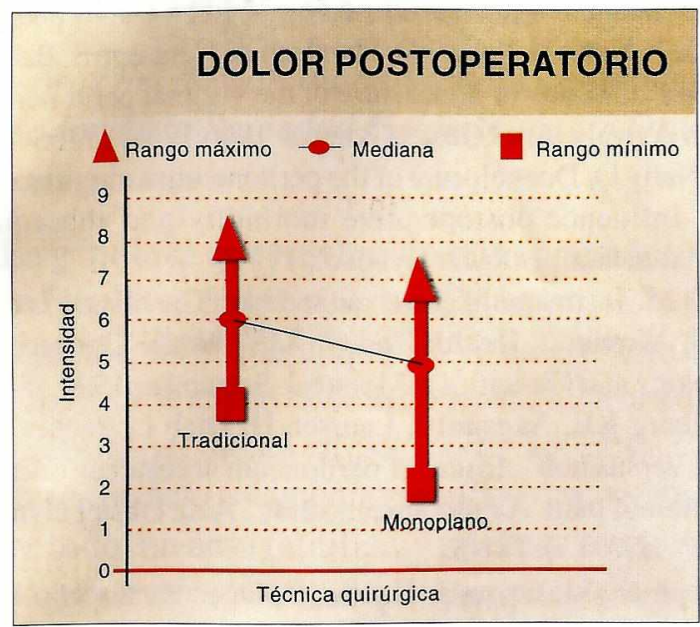

Figura 2. La intensidad del dolor postoperatorio en las pacientes a las que se les realizo la técnica tradicional fue mayor.

Con esta directriz de buscar el perfeccionamiento de una técnica quirúrgica que proporcionara seguridad tanto al cirujano como a la paciente (13), en 1926 Kerr describe la cesárea segmentaria (2).

En todo este proceso de evolución son muchas las técnicas que se han realizado. John C Hauth y colaboradores, de la Universidad de Alabama en Birmingham (2), realizaron un estudio similar al que se presenta, con una muestra ostensiblemente mayor $(n=906)$ en el que repartieron las pacientes en dos grupos: uno al que se le

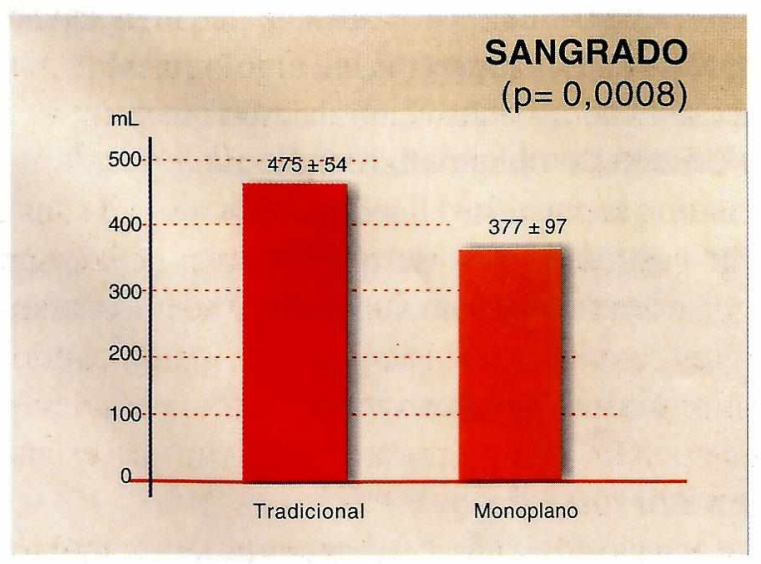

Figura 3. El promedio del sangrado intraoperatorio en el grupo manejado con la técnica de monoplano fue menor que el encontrado en las pacientes intervenidas con la técnica tradicional. realizó la histerorrafia en un solo plano $(n=457)$ y otro grupo $(n=449)$ de pacientes en quienes se realizó el cierre en dos planos. Las indicaciones de la cirugía fueron similares en ambos grupos.

Los autores encontraron una significativa reducción del tiempo quirúrgico en el grupo de un solo plano: 43,8 minutos vs 47,5 minutos en la técnica de biplano $(\mathrm{p}<0.001)$. Las infecciones, las complicaciones hemorrágicas y la estancia hospitalaria fueron similares en ambos grupos.

En el presente estudio no hubo complicaciones, pero deben tenerse en cuenta las diferencias en cuanto al tamaño de la muestra.

Llama la atención que entre los dos grupos de Hauth y colaboradores, un total de 369 pacientes (un plano $\mathrm{n}=179$ y dos planos $\mathrm{n}=190$ ) requirió puntos adicionales hemostáticos (2), mientras que en el estudio llevado a cabo en el Hospital de San José, un total de 24 pacientes requirió puntos adicionales ( $\mathrm{Gl} n=3$ y $\mathrm{G} 2 \mathrm{n}=23$ ).

Shelley y Owen del Departamentode GinecologíayObstetriciaen laUniversidad de Alabama en Birmingham, duranteel seguimiento del estudio anterior, lograron que 164 pacientes volvieran a lainstitución en el siguiente embarazo; deellas, a 83 se les había practicado cierre en un soloplano y a 81 en dos planos. De estas, a 19 se les realizó cesárea electiva y las otras 145 tuvieron parto vaginal.

Los resultados no mostraron diferencias significativas en cuanto al desarrollo del trabajo de parto y la duración de la estancia hospitalaria, como tampoco en otros parámetros tales como laincidenciadeadherencias, la infección puerperal, la hemorragiay la placentación anormal.

Este grupo de investigación concluye que el tipo de histerorrafia que se realice durante la cesárea no tiene efecto para el siguiente embarazo (13).

Para analizar el comportamiento de estas variables a largo plazo, se propone un estudio posterior de seguimiento.

Orla Mc Nally y colaboradores seleccionaron 100 pacientes de las cuales a 50 en la primera cesárea se les había realizado cierre de peritoneos y a las otras 50, no.

Los investigadores realizaron la segunda cesárea segmentaria y encontraron que la incidencia de adherencias fue mayor en el grupo al que se le había realizado cierre de peritoneo; $28 \%$ en comparación con sólo $14 \%$ en el grupo en el que no se cerró ( $\mathrm{p}<0.05)$. En dicho estudio concluyen que el cierre de peritoneos no es necesario y es uno de los factores que puede favorecer la formación de adherencias (10).

Hojberg y colaboradores no encontraron diferencias significativas con respecto al dolor postoperatorio en dos grupos que compararon, uno de los cuales correspondía 
a pacientes a quienes se les había realizado cierre de peritoneos $(\mathrm{n}=19)$ y el otro a quienes no $(\mathrm{n}=21)$; $\sin$ embargo, los requerimientos analgésicos fueron mayores en el grupo al cual se le había realizado el cierre de peritoneos (12).

En el grupo de estudio del Hospital de San José, el dolor postoperatorio fue más intenso en las pacientes a quienes se les realizó cierre de peritoneos.

Se sugiere la realización de un estudio de seguimiento de las pacientes, para evaluar las complicaciones a largo plazo y poder sustentar la implementación de la técnica con histerorrafia en monoplano sin cierre de peritoneos dentro de los protocolos de manejo del servicio, como una posible técnica segura y que reporta beneficios tanto para las pacientes como para la institución.

\section{Referencias}

1. Cunningham, Mac Donald, Gant Leveno. William's Obstetrics; $4^{\mathrm{a}}$ edición. Masson S.A., Barcelona, España, 585 -90.

2. Hauth JC, Owen J. Transverse Uterine Incision Closure : One versus two layer. Am J Obstet Gynecol. 1992 Oct; 167(4): 1108-11. 3. Federici D, Lacelly B. Cesarean Section using the Misgay Ladach method. Int J Gynaecol Obstet. 1997; 57(3): 273- 79. 4. Alonso P Walker. Peritoneo Peritonitis y Abscesos Intraabdominales. En: Schwars Principios de Cirugía General. Editorial MGH; 1303-1307.

5. Ellis H \& Reddle R. Does the Peritoneum to be closed at laparotomy. Brit J Surg, 1 1977; 164: 733-6.

6. Sanders, DiClementi. Principles of abdominal wound closure II. Prevention of wound dehiscence. Arch Surg. 1997; 112(10): 1188-91. 7. Hull DB, Varner NW. A randomized study of closure of the peritoneum at cesarean delivery, Obst Gynecol 1991; 77(6): 818-21.
8. Pietrantoni M, Parsons MT, O’Brien WF, Collins E, Knuppel RA, Spellacy WN. Peritoneal closure or non-closure at cesarean. Obstet Gynecol. 1991; 77(2):293-6.

9. Nagele F, Karas H, Spitzer D, Staudach A, Karasegh S, Beck A, Husslein P. Closure or nonclosure of the visceral peritoneum at cesarean delivery. Am J Obstet Gynecol. 1996;174(4):1366-70.

10. McNally O. Does closure of the peritoneum during cesarean section influence postoperative morbidity and subsequent bladder adhesion. J Obstet Gynecol, 1997.

11. Stark M. Technique of caesarean section: The Misgay Ladach method. Women's Health Today. XIV World Congress of Gynecology and Obstetrics at Montreal. September 1994. si - 85. 12. Hojberg KE, Aagaard J, Laursen H, Diab L, Secher NJ . Closure versus non -closure of peritoneum at cesarean section. Evaluation of pain. A randomized study . Acta Obstet Gynecol Scand. 1998;77 ( 7): 741-5.

13. Chapman SJ, Owen J, Hauth JC. One versus two-layer closure of a low transverse cesarean: the next pregnancy. Obstet Gynecol. 1997; 89: (1)16-8.

14. Tucker JM, Hauth JC, Hodgins P, Owen J, Winkler JL. Trial of labor after a one or two layer closure of a low transverse uterine incision. Am J Obstet Gynecol. 1993; 168(2): 545-6.

15. Obel J, Younis JS, Lang N, Levit A. Double-layer closure of uterine incision with visceral and parietal peritoneal closure: are they obligatory steps of routline cesarean sections? J Matern Fetal Med. 1996 Nov-Dec; 5(6): 366-9.

16. Karipineni RC, Wilk PJ, Danese CA. The role of the peritoneum in the healing of abdominal incisions. Surg Gyn Obstet. 1976; 142(5): 729-30.

17. Irion O, Luzuy F, Beguin F. Nonclosure of visceral and parietal peritoneum at cesarean section: a randomized controlled trial. Br J Obstet Gynaecol. 1996 Jul; 103(7): 690-4.

18) Pridjian G, Hibbard JU, Moawad AH. Cesarean: Changing the trends. Obstet Gynecol. 1991; 77(2): 195-200.

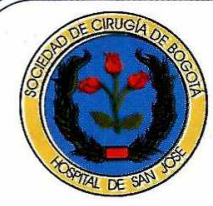

\section{SOCIEDAD DE CIRUGÍA DE BOGOTÁ \\ HOSPITAL DE SAN JOSÉ 1902 - 2000 HOSPITAL UNIVERSITARIO}

Atención integral del paciente, con énfasis en el manejo de alta complejidad, tecnología y excelente calidad de atención en cirugía y subespecialidades

- Área clínica y subespecialidades

- Pediatría y subespecialidades

- Servicios de apoyo diagnóstico: Resonancia nuclear magnética, Medicina nuclear, Tomografía axial computarizada, Banco de sangre, Laboratorio clínico y de inmunopatología 